\title{
Deviation of the Variances of Classical Estimators and Negative Integer Moment Estimator from Minimum Variance Bound with Reference to Maxwell Distribution
}

\author{
G. R. Pasha \\ Department of Statistics \\ Bahauddin Zakariya University \\ Multan, Pakistan \\ E-mail: drpasha@bzu.edu.pk \\ Muhammad Aslam \\ Department of Statistics \\ Bahauddin Zakariya University \\ Multan, Pakistan \\ E-mail: aslamasadi@bzu.edu.pk \\ Muhammad Javed \\ Department of Statistics \\ Bahauddin Zakariya University \\ Multan, Pakistan \\ E-mail: javedstat@yahoo.com
}

\begin{abstract}
In this paper, we present that how much the variances of the classical estimators, namely, maximum likelihood estimator and moment estimator deviate from the minimum variance bound while estimating for the Maxwell distribution. We also sketch this difference for the negative integer moment estimator. We note the poor performance of the negative integer moment estimator in the said consideration while maximum likelihood estimator attains minimum variance bound and becomes an attractive choice.
\end{abstract}

Keywords: Classical estimator; Cramer-Rao inequality; Maximum likelihood estimator; Maxwell distribution; Minimum variance bound; Moment estimator; Negative integer moment estimator.

\section{Introduction}

In the context of the kinetic molecular theory of gases, a gas contains a large number of particles in rapid motions. Each particle has a different speed, and each collision between particles changes the speeds of the particles. An understanding of the properties of the gas requires an understanding of the distribution of particle speeds. The Maxwell distribution describes the distribution of particle speeds in an ideal gas. This distribution has a variety of applications in the study of the distribution of speeds of molecules in thermal equilibrium as given by statistical mechanics (see Papoulis, 1984 for more details).

The probability density function of Maxwell distribution is given as

$$
f(x ; \theta)=\frac{\sqrt{2} x^{2}}{\theta^{3} \sqrt{\pi}} \exp \left(-\frac{x^{2}}{2 \theta^{2}}\right), \quad \text { for } \theta>0, x>0
$$


With the applications of the Maxwell distribution kinetic molecular theory of gases and a number of other similar cases, the estimation for its unknown parameter $\theta$ also become crucial. In the present paper, we compare two classical estimators, namely, maximum likelihood estimator (MLE) and estimator by method of moments (MME) with the negative integer moment estimator (NIME). Mohsin and Shahbaz (2005) also conducted such kind of comparison while estimating the parameter of inverse Rayleigh distribution but they just compare MLE with NIME. But in our study, in addition of MME, we mainly, compute minimum variance bound (MVB) for any unbiased estimator of unknown parameter of the Maxwell distribution and compute the deviation of this MVB form the variances of the said estimators.

In Section 2, we compute the classical estimates of the unknown value of the parameter $\theta$ for density (1.1) along with variances. For this we use MLE and MME. Section 3, dedicates for the estimation by method of negative integer moments. In Section 4, we give Cramer-Rao inequality and compute the MVB for estimation of $\theta$. In Section 5, we discuss the deviation of the variances from the MVB and relative efficiencies while Section 6 concludes.

\section{Classical Estimation}

The MLE is one of the classical estimators in statistical inference. Efron (1982) explained the method of maximum likelihood estimation along with the properties of the estimator. According to Aldrich (1997), the making of maximum likelihood was one of the most important developments in 20th century statistics. In his paper, he considered Fisher's changing justifications for the method.

It can be found that the log likelihood of (1.1) is;

$$
\log (L)=\frac{n}{2} \log \left(\frac{2}{\pi}\right)-3 n \log \theta+\log \left(\prod_{i=1}^{n} x_{i}^{2}\right)-\frac{1}{2 \theta^{2}} \sum_{i=1}^{n} x_{i}^{2},
$$

and, resultantly, the MLE of $\theta$ is given as

$$
\hat{\theta}_{M L}=\sqrt{\frac{\sum_{i=1}^{n} x_{i}^{2}}{3 n}},
$$

with

$$
\operatorname{var}\left(\hat{\theta}_{M L}\right)=\theta^{2}-\frac{2 \theta^{2}}{3 n}\left[\frac{\Gamma\left(\frac{3 n+1}{2}\right)}{\Gamma\left(\frac{3 n}{2}\right)}\right]^{2}
$$

The method of moment (MM) is also a commonly used method of estimation. In this method, the sample moments are assumed to be estimates of population moments and thus moment estimates for the unknown values of population parameters are found (see Lehman and Casella, 1998, for details).

For unknown parameter $\theta$ in (1.1), the moment estimator can be found as 


$$
\widehat{\theta}_{M M}=\frac{1}{2} \sqrt{\frac{\pi}{2}} \bar{X},
$$

where $\bar{X}$ is sample mean.

$$
\text { also, } \quad \operatorname{var}\left(\hat{\theta}_{M M}\right)=\frac{3 \pi-8}{8 n} \theta^{2}
$$

\section{Negative Integer Moment Estimator}

Negative integer moments are useful in applications in several contexts, notably in life testing problems. Bock et al. (1984) illustrated the examples of their use in the evaluation of a variety of estimators. With the particular reference to Chi-square distribution, in the inverse regression problem, Oman (1985) gave an exact formula for the mean squared error of Kruutchkoff's inverse estimator by use of negative integer moments of the noncentral Chi-squared variable.

The $r$ th negative integer moment is defined as:

$$
E\left\{(X+c)^{-r}\right\}
$$

where $X$ is a random variable, $\mathrm{c}$ is a constant, and $r$ is a positive integer.

Before finding the estimator by the method of negative integer moments, we have the $r$ th order negative integer moments of Maxwell distribution is as

$$
\mu_{(-r)}^{\prime}=\frac{2^{\frac{2-r}{2}}}{\sqrt{\pi}} \frac{1}{\theta^{r}} \Gamma\left(\frac{3-r}{2}\right)
$$

For $r=1$, the first order negative integer moment is;

$$
\mu_{(-1)}^{\prime}=\sqrt{\frac{2}{\pi}} \frac{1}{\theta}
$$

Now according to method of negative integer moment (NIM) estimation, the estimator for unknown parameter $\theta$ for (1.1) is

$$
\begin{aligned}
& \qquad \hat{\theta}_{N I M}=\sqrt{\frac{2}{\pi}} \frac{1}{m_{(-1)}^{\prime}}, \\
& \text { where } \quad m_{(-1)}^{\prime}=\frac{\sum_{i=1}^{n} 1 / x_{i}}{n} .
\end{aligned}
$$

For variance of estimator in (3.3), we have

$$
\operatorname{var}\left(\hat{\theta}_{N I M}\right)=\frac{\pi-2}{2 n} \theta^{2}
$$

\section{Minimum Variance Bound}

Let $X=\left(X_{1}, X_{2}, \ldots, X_{n}\right)$ be a random sample and let $f(x ; \theta)$ denotes the probability density function for some model of the data with unknown parameter $\theta$. If $T(X)$ be 
any statistic and $\psi(\theta)$ be its expectation such that $\psi(\theta)=E[T(X)]$. Under some regularity conditions (see Lehman and Casella, 1998), it follows that for all $\theta$,

$$
\operatorname{Var}(T(X)) \geq \frac{\left(\frac{\partial \psi(\theta)}{\partial \theta}\right)^{2}}{I_{n}(\theta)},
$$

where $I_{n}(\theta)$ is Fisher's information matrix.

This is called Cramer-Rao inequality and the right hand side of (4.1) is called Cramer-Rao lower bound or MVB. In particular, if $T(X)$ is an unbiased estimator of $\theta$, then the numerator becomes 1 , and MVB is simply $1 / I_{n}(\theta)$. Obviously, if the variance of estimator coincides with the MVB, it means that we are using an estimator that bears minimum variance in its class of estimators.

For the unknown parameter in (1.1), it can easily be shown that for any unbiased estimator of $\theta$,

$$
\mathrm{MVB}=\frac{\theta^{2}}{6 n}
$$

\section{Deviation from MVB and Relative Efficiency}

We compare all the three variances (2.3), (2.5), and (3.4) with the MVB given in (4.2). Since all these variances contains the term $\theta^{2}$ as a multiplier so we drop it while comparing them all. Fig. 5.1 shows the deviation of the variances (actual variances in the figure should be multiplied by $\theta^{2}$ ) of the estimators under consideration from the MVB. These values are drawn against different sample sizes. We note that the variance of MLE has least deviation from the MVB. As the sample size increases, the variance of MLE begins to coincide with the MVB. For $n>20$, variance of MLE equalizes to the MVB.

For small samples, the variance of moment estimator notably deviates from the MVB but this difference covers with the increase in sample size. When sample size becomes larger than 30 , this variance begins to coincide with the MVB.

In the case of NIM estimation, the performance of NIME remains quite miserable, especially, for small sample. The variance of NIME remains largely deviant from the MVB as it falls miles away form the MVB. Although, the severity of the deviation decreases with the increase in sample size but even then it does not appear to make NIME attractive while comparing with its two competitors.

As for as relative efficiency is concerned, we note the efficiency of MLE relative to NIME as 3.45 for $n=10$ and MLE remains about 2.5 times more efficient as compared to NIME for larger sample size. Our findings thus also matches to those of Mohsin and Shahbaz (2005) who also declared MLE more efficient as compared to NIME while estimating the parameter of inverse Rayleigh distribution. 
We also note that MME remains about 2.2 times more efficient as compared to NIME. We further note that this relative efficiency dose not change with the increase in sample size. When we compare MLE with MME for variances, we find MLE about 7\% more efficient as compared to the moment estimator.

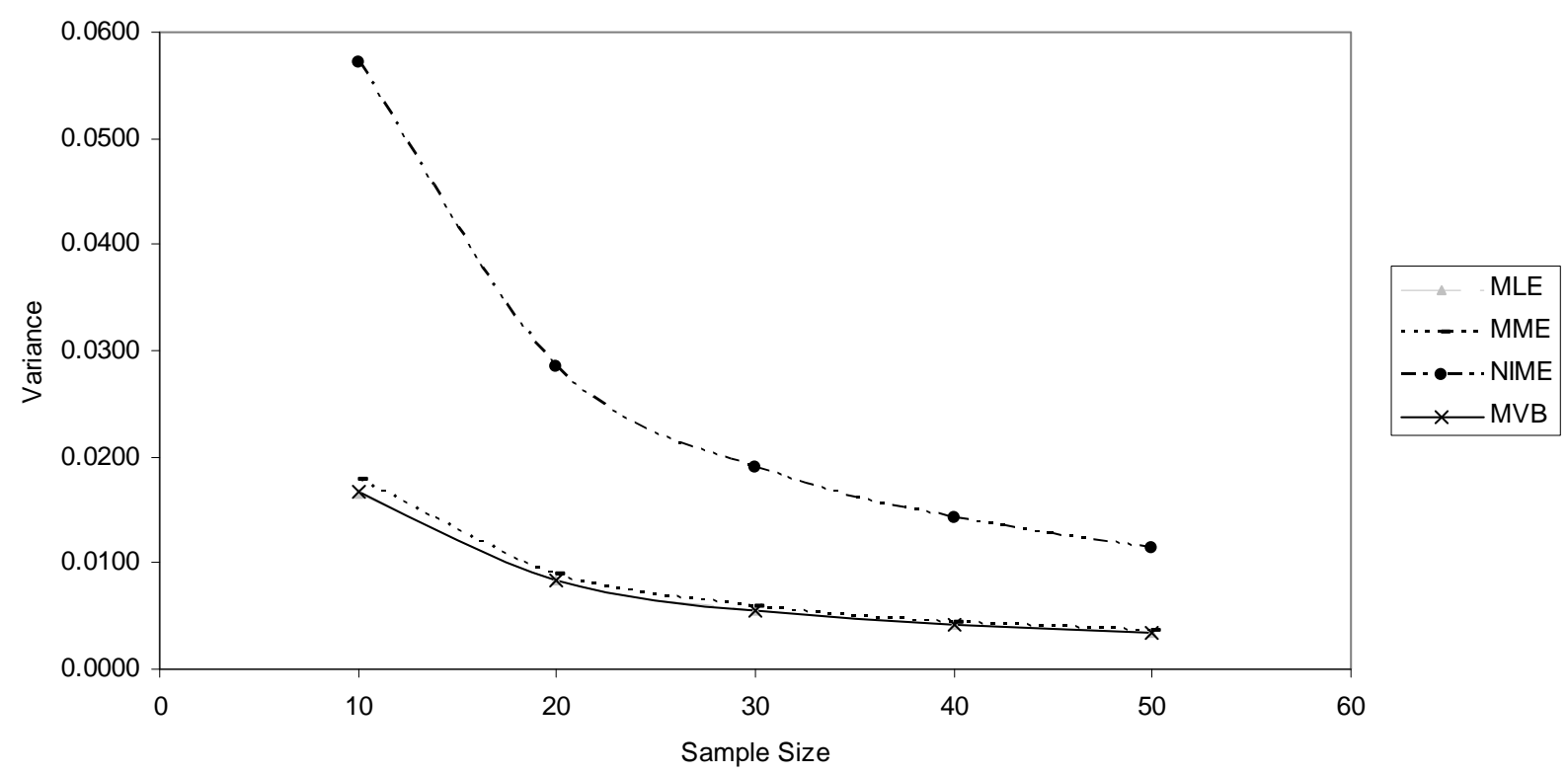

Figure 5.1: Deviation from MVB

\section{Conclusion}

Although the negative integer moments are gaining popularity for their applications but NIM estimator performs poorly in the term of efficiency while estimating the unknown parameter of the Maxwell distribution. Its variance remain too far form the Cramer-Rao lower bound for variance i.e., MVB. When we are dealing with the kinetic molecular theory of gases or the matters of thermodynamic with the application of the Maxwell distribution, MLE is the best choice for estimating the unknown parameter. Even for large samples $(n>30)$, moment estimator may equally be utilized.

\section{References}

1. Aldrich, J. (1997). R. A. Fisher and the Making of Maximum Likelihood 1912 - 1922. Statistical Science, 3, 162-176.

2. Bock, M. E., Judge, G. G. and Yancy, T. A. (1984). A Simple Form for the Inverse Moments of Non-central Chi-Square and F Random Variables and Certain Confluent Hypergeometric Functions. J. Econometrics, 25, 217234.

3. Efron, B. (1982). Maximum Likelihood and Decision Theory. Ann. Statist. 10, 340-356.

4. Lehman, E. L., and Casella, G. (1998). Theory of Point Estimation. Springer, New York. 


\section{G. R. Pasha, Muhammad Aslam, Muhammad Javed}

5. Mohsin, M. and Shahbaz, M.Q., (2005). Comparison of Negative Moment Estimator with Maximum Likelihood Estimator of Inverse Rayliegh Distribution, PJSOR, 1, 45-48

6. Oman, S. D., (1985). An Explicit Formula for the Mean Squared Error of the Inverse Estimator in the Linear Calibration Problem, Journal of Statistics Planning and Inference, 11, 189-19

7. Papoulis, A. (1984). Probability, Random Variables, and Stochastic Processes. McGraw-Hill, New York. 